\title{
Composição corporal em crianças de sete a 10 anos de idade, de alto nível socioeconômico
}

\author{
Enio Ricardo Vaz Ronque 1,2,3, Débora Alves Guariglia', Edilson Serpeloni Cyrino ${ }^{1,2}$,
} Ferdinando Oliveira Carvalho', Ademar Avelar ${ }^{1}$ e Miguel de Arruda1,2,3

\section{RESUMO}

O objetivo deste estudo foi analisar a composição corporal de crianças de sete a 10 anos de idade, de alto nível socioeconômico. Para tanto, 511 crianças (274 meninos e 237 meninas) foram submetidas a medidas antropométricas de massa corporal, estatura e espessuras de dobras cutâneas tricipital (TR) e subescapular (SE). Com base nessas informações, foram determinados: gordura corporal relativa (\% Gordura), massa corporal magra (MCM), distribuição da gordura corporal (DGC) e o somatório da espessura das dobras cutâneas TR e SE ( $\Sigma E D C)$. Anova two-way foi utilizada para as comparações entre os sexos e os diferentes grupos etários, seguida pelo teste post hoc de Scheffé quando $P<0,05$. Para a classificação das crianças, de acordo com as categorias de adiposidade corporal, foi utilizada distribuição de freqüência. O teste de comparação entre proporções foi adotado para verificar as diferenças entre os sexos, em cada categoria $(P<0,05)$. Diferenças significantes entre os sexos $(P<0,05)$ foram verificadas na dobra TR (meninas > meninos) e na MCM (meninos > meninas). Efeito significante da idade foi identificado em todas as variáveis analisadas, exceto na DGC, com os valores mais elevados sendo encontrados no grupo etário de 10 anos. Um percentual elevado, tanto de meninos quanto de meninas, apresentou índice de adiposidade alto, com superioridade para os meninos (28\% vs. $14 \%, P<$ $0,01)$. Por outro lado, um contingente maior de meninas apresentou concentrações de gordura corporal relativa abaixo dos pontos de corte desejáveis $(15 \%$ vs. $3 \%, P<0,01)$. Os valores de adiposidade corporal encontrados no presente estudo sugerem que um percentual elevado de crianças, de ambos os sexos, já apresenta importantes fatores de risco à saúde, em idades precoces.

\section{ABSTRACT}

\section{Body composition in seven-to-ten-year old children of high socioeconomic status}

The objective of this study was to analyze the body composition of seven to 10 year-old children of high socioeconomic status. Therefore, 511 children (274 boys and 237 girls) were submitted to body mass, height and triceps (TR) and subscapular (SE) skinfolds thickness anthropometric measurement. Based on this information, the following topics were determined: relative body fat (\% Fat), lean body mass (LBM), body fat distribution (BFD) and sum of the thickness of the TR and SE skinfolds (ETSF). Two-way ANOVA was used for comparisons between gender and the dif-

1. Grupo de Estudo e Pesquisa em Metabolismo, Nutrição e Exercício, Centro de Educação Física e Esporte, Universidade Estadual de Londrina.

2. Grupo de Estudo e Pesquisa em Atividade Física e Exercício, Centro de Educação Física e Esporte, Universidade Estadual de Londrina.

3. Faculdade de Educação Física, Universidade Estadual de Campinas. Aceito em 13/6/07.

Endereço para correspondência: Enio Ricardo Vaz Ronque, Rua Serra do Roncador, 307, Casa 135, Jardim Bandeirantes - 86065-590 - Londrina, PR. E-mail: enioronque@sercomtel.com.br

\begin{abstract}
Palavras-chave: Gordura corporal. Antropometria. Baixo peso. Sobrepeso. Obesidade.

Keywords: $\quad$ Body fat. Anthropometric measurement. Anthropometry. Low weight. Overweight. Obesity.
\end{abstract}

ferent age groups, followed by the Scheffé post hoc test when $P$ $<0.05$. For the children classification according to the categories of body adiposity, frequency distribution was used. A test of comparison between ratios was adopted to verify the differences between gender in each category $(P<0.05)$. Significant differences between gender $(P<0.05)$ were verified in TR skinfold (girls > boys) and in LBM (boys > girls). Significant age effect was identified in all analyzed variables, except for DBF, with the highest values having been found in the 10 year-old group. A high percentage both for boys and girls presented high adiposity index, with superiority for boys (28\% vs. $14 \%, P<0.01)$. On the other hand, a larger number of girls presented relative body fat concentrations below the desirable cutting points $(15 \%$ vs. $3 \%, P<0.01)$. The values of body adiposity found in the present study suggest that a high percentage of children from both gender already present important health risk factors at early ages.

\section{INTRODUÇÃO}

Durante o período da infância e da adolescência pode ser observado um aumento gradativo tanto da massa de gordura quanto da massa corporal magra. Assim, o monitoramento dessas variáveis pode favorecer a identificação de problemas de saúde associados aos níveis reduzidos de gordura corporal(1) ou a elevados índices de adiposidade ${ }^{(2-3)}$.

Embora a prevalência de sobrepeso/obesidade em indivíduos de ambos os sexos, em diferentes faixas etárias, em diversos países do mundo, possa ser considerada um fenômeno mundial e de natureza multifatorial, a redução nos níveis de atividade física habitual e os hábitos nutricionais inadequados, de forma isolada ou combinada, parecem ser os principais fatores determinantes da chamada epidemia do sobrepeso/obesidade que se verifica atualmente ${ }^{(4)}$. O que tem chamado a atenção, sobretudo, dos pesquisadores e profissionais das áreas de saúde, é que esse fenômeno tem atingido um contingente cada vez maior de crianças e adolescentes, podendo gerar diversos transtornos a saúde, em idades precoces.

Vale ressaltar que cerca de $50 \%$ dos sujeitos obesos durante a adolescência tornam-se adultos obesos e que aproximadamente $1 / 3$ dos adultos obesos já tinham esse quadro instalado durante a infância(5). As conseqüências da obesidade pediátrica para o estado adulto estão relacionadas não somente com a quantidade da gordura corporal, mas, em particular, a sua distribuição pelo corpo, bem como a permanência da sobrecarga ponderal patológica até o final da adolescência(6-7).

Níveis elevados de gordura corporal acompanhados de uma distribuição centralizada são importantes fatores de risco para o desenvolvimento de doença arterial coronariana, independentemente das quantidades de gordura visceral(7-9). Vale destacar que a 
distribuição centrípeta do tecido adiposo pode contribuir, também, para o aparecimento de dislipidemias, comprometer o metabolismo das lipoproteínas plasmáticas e dificultar a manutenção da pressão arterial em níveis satisfatórios ${ }^{(10)}$.

Desse modo, o sobrepeso/obesidade durante a infância e adolescência favorece o aumento da prevalência de síndrome metabólica na idade adulta(11) e possui estreita relação com o desenvolvimento prematuro da aterosclerose ${ }^{(12)}$, contribuindo sobremaneira para o aumento das taxas de mortalidade cardiovascular ${ }^{(13)}$.

Em face dessa situação, o diagnóstico precoce do sobrepeso/ obesidade tem sido uma das prioridades em saúde pública ${ }^{(14)}$, uma vez que a normalização ponderal durante a infância e a adolescência pode minimizar o risco de morbidade/mortalidade na idade adul$\mathrm{ta}^{(9)}$.

Considerando as desigualdades sociais e as diversidades culturais observadas no Brasil, a análise da composição corporal de populações jovens, de diferentes níveis socioeconômicos, pode proporcionar valiosas informações para o desenvolvimento de políticas públicas de educação para a saúde. Portanto, o propósito deste estudo foi analisar a composição corporal de crianças de sete a 10 anos de idade, de alto nível socioeconômico.

\section{METODOLOGIA}

\section{Sujeitos}

As informações apresentadas neste estudo fazem parte da coleta de dados inicial de um projeto de pesquisa mais amplo, de caráter longitudinal, intitulado "Análise do crescimento e da aptidão física relacionada à saúde em escolares de alto nível socioeconômico", que vem sendo realizado no município de Londrina (PR), Brasil.

A população alvo deste estudo foi composta por escolares de sete a 10 anos de idade, de ambos os sexos, de alto nível socioeconômico, residentes na área urbana desse município.

Um levantamento preliminar, para identificação do número de escolares matriculados na rede particular de ensino desse município, no ano letivo de 2002, foi realizado antes da seleção da amostra. De acordo com o setor de estatística do Núcleo Regional de Ensino da Secretaria de Educação do Estado do Paraná, estavam matriculados, somente na região urbana, 15.778 estudantes, sendo 1.551 no ensino pré-escolar $(9,8 \%)$; 3.874 no primeiro e segundo ciclos do ensino fundamental I $(24,0 \%) ; 4.134$ no terceiro e quarto ciclos do ensino fundamental II $(26,2 \%)$; 3.976 no ensino médio $(25,2 \%)$; 46 no ensino normal $(0,3 \%) ; 1.857$ no ensino técnico $(11,8 \%)$ e 430 no ensino supletivo $(2,7 \%)$.

Com base nessas informações uma escola da região central foi selecionada de forma intencional, uma vez que atendia aos critérios estabelecidos para o desenvolvimento do presente estudo quanto ao nível socioeconômico, possuía um número expressivo de escolares matriculados na rede particular de ensino do município ( 15\%) e apresentava infra-estrutura adequada para a coleta de dados.

Dessa forma, a amostra do presente estudo foi composta por 511 escolares, sendo 274 meninos e 237 meninas, entre sete e 10 anos de idade, de alto nível socioeconômico, matriculados na rede particular de ensino do município de Londrina, PR, Brasil. Os grupos etários foram estabelecidos em idades decimais conforme os procedimentos descritos por Ross e Marfell-Jones ${ }^{(15)}$. Para o agrupamento por idade, para cada sexo, foram utilizados os intervalos de 0,50 a 0,49 convencionado com o sinal \pm , de acordo com Eveleth e Tanner ${ }^{(16)}$.

Este estudo foi desenvolvido em conformidade com as instruções contidas na Resolução 196/96 do Conselho Nacional de Saúde para estudos com seres humanos, do Ministério da Saúde, sendo aprovado pelo Comitê de Ética em Pesquisa da Universidade Estadual de Londrina. Todos os responsáveis pelos escolares, após serem informados sobre o propósito desta investigação e os procedimentos a serem adotados, assinaram um termo de consentimento livre e esclarecido. A distribuição da amostra, de acordo com sexo e idade, é apresentada na tabela 1.

TABELA 1

Distribuição da amostra de acordo com sexo e idade

\begin{tabular}{lccc}
\hline \multicolumn{1}{c}{ Grupo etário } & Meninos & Meninas & Total \\
\pm 7 anos $(7,18 \pm 0,18)$ & 38 & 36 & 74 \\
\pm 8 anos $(8,01 \pm 0,29)$ & 86 & 61 & 147 \\
\pm 9 anos $(8,99 \pm 0,29)$ & 65 & 64 & 129 \\
\pm 10 anos $(10,01 \pm 0,29)$ & 85 & 76 & 161 \\
Total & 274 & 237 & 511 \\
\hline
\end{tabular}

Nota: As informações entre parênteses expressam valores médios ( \pm DP).

Para classificação do nível socioeconômico foi aplicado um questionário desenvolvido pela ABA/ABIPEME e adaptado por Almeida e Wickerhauser(17), com escalas de classificação subdivididas em cinco categorias ( $A, B, C, D, E)$, de acordo com o grau de instrução dos pais e os bens de consumo familiar. Com base nesse instrumento, todos os participantes foram classificados como pertencentes ao nível socioeconômico elevado (categorias A e B).

\section{Antropometria}

A massa corporal dos sujeitos foi obtida em uma balança digital, da marca Filizola, com precisão de 0,05kg, e a estatura foi determinada em um estadiômetro de madeira, com precisão de $0,1 \mathrm{~cm}$, conforme os procedimentos descritos por Gordon et al.(18). A partir das medidas de massa corporal e estatura calculou-se o índice de massa corporal (IMC) por meio do quociente massa corporal/estatura ${ }^{2}$, sendo a massa corporal expressa em quilogramas $(\mathrm{kg})$ e a estatura em metros $(\mathrm{m})$.

Para a avaliação do comportamento da adiposidade subcutânea foram medidas as espessuras das dobras cutâneas tricipital (TR) e subescapular (SE). Tais medidas foram realizadas por um único avaliador, com um adipômetro científico da marca Lange (Cambridge Scientific Industries, Inc., Cambridge, Maryland), de acordo com os procedimentos descritos por Harrison et al. ${ }^{(19)}$. O coeficiente teste-reteste excedeu 0,95 para cada um dos pontos anatômicos, com erro de medida de no máximo $\pm 1,0 \mathrm{~mm}$. Todas as medidas foram tomadas de forma rotacional e replicadas três vezes, sendo registrado o valor mediano. Com base nos valores das espessuras de dobras cutâneas TR e SE, os resultados foram interpretados isoladamente, bem como pelo somatório da espessura dessas duas dobras cutâneas ( $E \mathrm{EDC})$, com os resultados sendo expressos em milímetros ( $\mathrm{mm}$ ).

A gordura corporal relativa (\%Gordura) foi estimada por meio das equações propostas por Slaughter et al.(20), e o padrão de distribuição da gordura corporal (DGC) foi determinado mediante a relação entre as espessuras das dobras cutâneas de tronco (SE) e de membros (TR). Os valores de massa corporal magra (MCM) foram determinados pela simples subtração entre a massa corporal e a quantidade de gordura absoluta.

A partir dos valores de \%Gordura a amostra foi classificada, de acordo com as categorias de adiposidade propostas por Lohman (1) (tabela 2).

\begin{tabular}{lcc}
\hline & $\begin{array}{c}\text { TABELA } 2 \\
\text { Classificação do índice de adiposidade de } \\
\text { acordo com a gordura corporal relativa (\%) }\end{array}$ & \\
\hline Classificação & Meninos & Meninas \\
Baixo & $<10,0$ & $<15,0$ \\
Ótimo & $\geq 10,0$ e $\leq 20,0$ & $\geq 15,0$ e $\leq 25,0$ \\
Moderadamente alto & $>20,0$ e $<25,0$ & $>25,0$ e $<30,0$ \\
Alto & $\geq 25,0$ & $\geq 30,0$ \\
\hline
\end{tabular}

Nota: Adaptado de Lohman (20). 


\section{Tratamento estatístico}

O teste de normalidade de Kolmogorov-Smirnov foi utilizado para análise da distribuição dos dados. Para as comparações entre os diferentes grupos etários, e entre os sexos, foi empregada análise de variância por dois fatores (Anova two-way), seguida pelo teste post hoc de Scheffé, quando $P<0,05$. Para a classificação das crianças de acordo com as categorias de adiposidade foi utilizada distribuição de freqüência. O teste de comparação entre proporções foi adotado para identificar possíveis diferenças entre os sexos, em cada categoria de adiposidade $(P<0,05)$. As informações foram processadas nos pacotes computacionais Statistica ${ }^{\mathrm{TM}}$ e MedCalc.

\section{RESULTADOS}

Os valores de massa corporal, estatura e IMC da amostra investigada são apresentados na tabela 3 , de acordo com sexo e grupo etário.

\section{TABELA 3}

Valores médios ( \pm DP) da massa corporal, estatura e índice de massa corporal (IMC) em crianças de ambos os sexos

\begin{tabular}{|c|c|c|c|c|c|c|}
\hline \multirow{2}{*}{$\begin{array}{l}\text { Idade } \\
\text { (anos) }\end{array}$} & \multicolumn{2}{|c|}{ Massa corporal (kg) } & \multicolumn{2}{|c|}{ Estatura (cm) } & \multicolumn{2}{|c|}{ IMC $\left(\mathbf{k g} / \mathbf{m}^{2}\right)$} \\
\hline & Meninos & Meninas & Meninos & Meninas & Meninos & Meninas \\
\hline \pm 7 & $25,6 \pm 4,0$ & $26,6 \pm 5,3$ & $123,3 \pm 3,7$ & $125,6 \pm 4,7$ & $16,7 \pm 2,0$ & $16,7 \pm 2,5$ \\
\hline \pm 8 & $29,7 \pm 7,1$ & $28,2 \pm 6,5$ & $130,1 \pm 6,2$ & $127,4 \pm 5,9$ & $17,3 \pm 2,9$ & $17,2 \pm 2,8$ \\
\hline \pm 9 & $34,9 \pm 9,5$ & $31,5 \pm 7,1$ & $135,3 \pm 7,0$ & $133,6 \pm 6,5$ & $18,8 \pm 4,0$ & $17,5 \pm 2,9$ \\
\hline \pm 10 & $37,5 \pm 8,4$ & $35,8 \pm 7,5$ & $140,0 \pm 6,4$ & $140,2 \pm 7,0$ & $18,9 \pm 3,1$ & $18,1 \pm 2,9$ \\
\hline Fsexo & \multicolumn{2}{|c|}{$4,10 * *$} & \multicolumn{2}{|c|}{0,72} & \multicolumn{2}{|c|}{$4,28 * *$} \\
\hline Fidade & \multicolumn{2}{|c|}{$46,16^{*}$} & \multicolumn{2}{|c|}{$139,46^{*}$} & \multicolumn{2}{|c|}{$8,17^{*}$} \\
\hline $\begin{array}{l}\text { Fsexo } \\
x \text { idade }\end{array}$ & \multicolumn{2}{|c|}{1,39} & \multicolumn{2}{|c|}{$3,18 * *$} & \multicolumn{2}{|c|}{1,29} \\
\hline
\end{tabular}

* $P \leq 0,01$ e ${ }^{*} 0,01<P<0,05$.

Na tabela 4 são apresentados os valores de média e desvio padrão e da análise de variância (Anova) para a espessura de dobras cutâneas da região subescapular (SE) e tricipital (TR), bem como para a somatória das espessuras das dobras cutâneas (TR + SE), de acordo com sexo e a idade.

\section{TABELA 4}

Valores médios (ะ DP) das espessuras de dobras cutâneas subescapular (SE) e tricipital (TR) e do $\Sigma E D C$ em crianças de ambos os sexos

\begin{tabular}{|c|c|c|c|c|c|c|}
\hline \multirow{2}{*}{$\begin{array}{l}\text { Idade } \\
\text { (anos) }\end{array}$} & \multicolumn{2}{|c|}{ SE (mm) } & \multicolumn{2}{|c|}{$\mathrm{TR}(\mathrm{mm})$} & \multicolumn{2}{|c|}{$\Sigma E D C(\mathrm{~mm})$} \\
\hline & Meninos & Meninas & Meninos & Meninas & Meninos & Meninas \\
\hline \pm 7 & $7,6 \pm 3,4$ & $8,7 \pm 4,2$ & $11,1 \pm 3,3$ & $13,0 \pm 4,3$ & $18,7 \pm 6,3$ & $21,7 \pm 8,2$ \\
\hline \pm 8 & $8,4 \pm 5,0$ & $9,1 \pm 4,7$ & $12,0 \pm 5,1$ & $13,5 \pm 3,9$ & $20,4 \pm 9,7$ & $22,7 \pm 8,2$ \\
\hline \pm 9 & $11,3 \pm 7,6$ & $10,6 \pm 5,9$ & $15,1 \pm 6,8$ & $14,9 \pm 5,3$ & $26,5 \pm 14,0$ & $25,5 \pm 10,8$ \\
\hline \pm 10 & $10,7 \pm 6,5$ & $11,5 \pm 6,3$ & $15,1 \pm 6,0$ & $16,5 \pm 5,2$ & $25,9 \pm 12,0$ & $28,1 \pm 10,8$ \\
\hline Fsexo & \multicolumn{2}{|c|}{0,88} & \multicolumn{2}{|c|}{$5,25^{* *}$} & \multicolumn{2}{|c|}{2,72} \\
\hline Fidade & \multicolumn{2}{|c|}{$7,65^{*}$} & \multicolumn{2}{|c|}{$13,70^{*}$} & \multicolumn{2}{|c|}{$11,16^{*}$} \\
\hline $\begin{array}{l}\text { Fsexo } \\
\mathrm{x} \text { idade }\end{array}$ & \multicolumn{2}{|c|}{0,60} & \multicolumn{2}{|c|}{0,90} & \multicolumn{2}{|c|}{0,79} \\
\hline
\end{tabular}

Nota: $\Sigma$ EDC = somatório das espessuras das dobras cutâneas subescapular e tricipital.

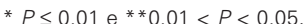

O efeito isolado da idade foi constatado na SE $(P<0,01)$, sendo que tanto meninos quanto meninas apresentaram um aumento progressivo com o avançar da idade. Para a TR, além do efeito da idade $(P<0,01)$, verificou-se também o efeito do sexo $(P<0,05)$, com as meninas apresentando valores superiores aos meninos, apesar de nenhuma diferença significativa ter sido encontrada dentro do mesmo grupo etário.
Um comportamento semelhante ao observado na SE foi verificado no $\Sigma$ EDC, ou seja, somente o efeito da idade $(P<0,01)$ foi identificado, com valores crescentes, em ambos os sexos. Portanto, na SE o aumento com o avançar da idade foi na ordem de aproximadamente $40 \%$ para os meninos e $32 \%$ para as meninas, ao passo que na TR foi de $36 \%$ e $27 \%$, respectivamente, para meninos e meninas. Desse modo, um acréscimo de 39\% no $\Sigma$ EDC foi constatado nos meninos, bem como de $30 \%$ nas meninas.

Informações sobre a gordura corporal relativa (\% Gordura), massa corporal magra (MCM) e distribuição da gordura corporal (DGC), de acordo com o sexo e a idade, são apresentadas na tabela 5.

TABELA 5

Valores médios (ะ DP) de gordura corporal relativa (\%Gordura), de massa corporal magra (MCM) e da distribuição de gordura corporal (DGC) em crianças de ambos os sexos

\begin{tabular}{|c|c|c|c|c|c|c|}
\hline \multirow{2}{*}{$\begin{array}{l}\text { Idade } \\
\text { (anos) }\end{array}$} & \multicolumn{2}{|c|}{ \%Gordura } & \multicolumn{2}{|c|}{ MCM (kg) } & \multicolumn{2}{|c|}{ DGC (mm) } \\
\hline & Meninos & Meninas & Meninos & Meninas & Meninos & Meninas \\
\hline \pm 7 & $17,8 \pm 5,2$ & $19,5 \pm 5,8$ & $20,8 \pm 2,2$ & $21,1 \pm 3,0$ & $0,69 \pm 0,28$ & $0,66 \pm 0,15$ \\
\hline \pm 8 & $18,9 \pm 7,5$ & $20,3 \pm 5,7$ & $23,6 \pm 3,3$ & $22,2 \pm 3,6$ & $0,68 \pm 0,17$ & $0,66 \pm 0,18$ \\
\hline \pm 9 & $23,4 \pm 10,7$ & $22,1 \pm 7,1$ & $25,8 \pm 4,0$ & $24,1 \pm 3,5$ & $1,72 \pm 0,19$ & $0,69 \pm 0,17$ \\
\hline \pm 10 & $22,4 \pm 9,4$ & $23,9 \pm 6,9$ & $28,5 \pm 4,2$ & $26,8 \pm 3,8$ & $0,69 \pm 0,17$ & $0,68 \pm 0,21$ \\
\hline Fsexo & \multicolumn{2}{|c|}{1,42} & \multicolumn{2}{|c|}{$10,92 *$} & \multicolumn{2}{|c|}{1,41} \\
\hline Fidade & \multicolumn{2}{|c|}{$9,50 *$} & \multicolumn{2}{|c|}{$71,35^{*}$} & \multicolumn{2}{|c|}{0,56} \\
\hline $\begin{array}{l}\text { Fsexo } \\
\text { x idade }\end{array}$ & \multicolumn{2}{|c|}{1,01} & \multicolumn{2}{|c|}{1,48} & \multicolumn{2}{|c|}{0,14} \\
\hline
\end{tabular}

${ }^{*} P \leq 0,01 e^{* *} 0,01<P<0,05$.

Na variável DGC não foram identificados efeitos que pudessem ser atribuídos ao sexo e a idade $(P>0,05)$. O efeito do sexo foi verificado somente na $\mathrm{MCM}(P<0,01)$, com os meninos apresentando valores superiores aos das meninas. Por outro lado, o efeito da idade foi constatado no \%Gordura e na $\operatorname{MCM}(P<0,01)$, com os valores crescentes acompanhando o avanço da idade, em ambos os sexos.

O \% Gordura foi aproximadamente $26 \%$ e $23 \%$ maior, em meninos e meninas, respectivamente, dos sete aos 10 anos, ao passo que a MCM foi $37 \%$ maior nos meninos e $27 \%$ nas meninas, no mesmo período. Apesar disso, diferenças significativas entre os sexos não foram verificadas $(P>0,05)$, dentro do mesmo grupo etário.

A figura 1 apresenta a classificação dos sujeitos, de acordo com as diferentes categorias de adiposidade. Pouco mais da metade da amostra de meninos e meninas ( $55 \%)$ apresentaram valores

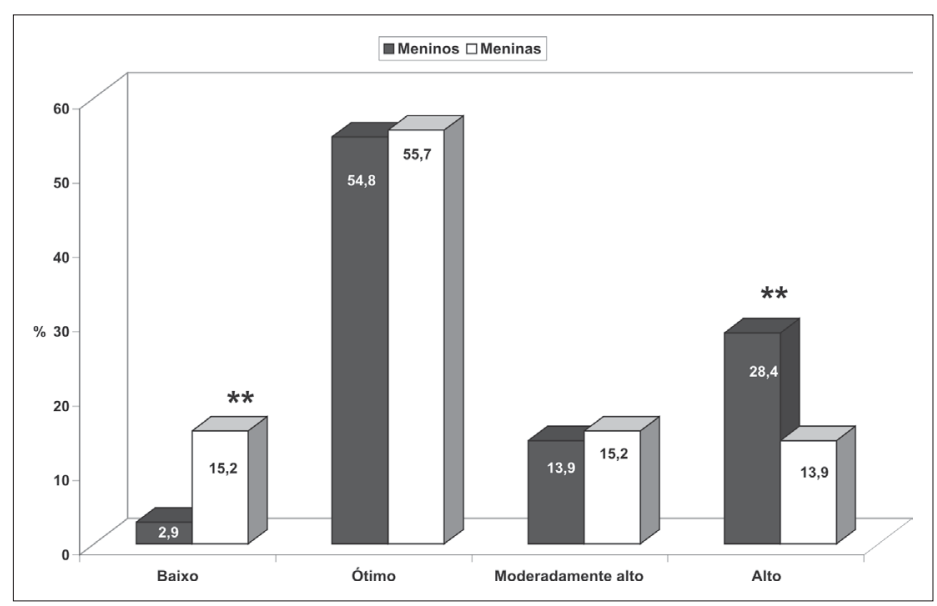

Figura 1 - Comparações entre meninos e meninas, de acordo com a classificação estabelecida a partir dos valores do índice de adiposidade Nota: Os resultados de cada categoria são representativos dos valores percentuais encontrados para cada sexo

** $P \leq 0,01$. 
desejáveis de adiposidade, todavia sem diferenças estatisticamente significantes entre os sexos $\left(\chi^{2}=0,013 ; P=0,9110\right)$.

Os resultados indicam que por volta de $14 \%$ e $15 \%$, dos meninos e das meninas, respectivamente, apresentam risco de obesidade $\left(\chi^{2}=0,084 ; P=0,7761\right)$, ao passo que um maior contingente de meninos apresentou concentrações excessivamente altas de adiposidade do que as meninas ( $28 \%$ vs. $14 \% ; \chi^{2}=14,896 ; P<$ $0,001)$. Por outro lado, um maior número de meninas $(15 \%)$ do que meninos ( $3 \%)$ apresentaram baixos índices de adiposidade $\left(\chi^{2}=22,908 ; P=0,0001\right)$.

\section{DISCUSSÃo}

O interesse pelo estudo da composição corporal em crianças e jovens reside no fato de ser possível obter informações sobre o fracionamento da massa corporal em seus diferentes componentes, uma vez que a quantidade e a distribuição da gordura corporal apresentam uma estreita relação com alguns indicadores de saúde.

Portanto, se por um lado baixos índices de adiposidade corporal podem indicar possíveis deficiências nutricionais ${ }^{(21)}$, por outro lado o acúmulo excessivo de gordura corporal, via de regra, pode indicar um desequilíbrio entre o consumo e a demanda energética diária. Nesse sentido, o acúmulo excessivo de gordura corporal está associado ao desenvolvimento ou agravamento de inúmeras disfunções metabólicas, tais como doenças cardiovasculares, hipertensão, diabetes, câncer, entre tantas outras(22-24). Além disso, uma maior adiposidade corporal verificada na infância associa-se com a preservação desse comportamento na idade adulta(24)

Verificou-se no presente estudo que o efeito da idade sobre o comportamento das espessuras das dobras cutâneas nas crianças investigadas foi similar ao relatado por outros estudos ${ }^{(26-30)}$. A inexistência de diferenças estatisticamente significantes entre os sexos, dentro do mesmo grupo etário, na SE pode estar associada à hipótese de que o dimorfismo sexual ocorre somente a partir do início da puberdade, tanto para variáveis da composição corporal quanto para aquelas relacionadas ao crescimento físico(30).

Por outro lado, ao se analisar os resultados obtidos nesta investigação pode-se verificar que os valores médios encontrados para as espessuras de dobras utilizadas foram similares ${ }^{(27,30)}$ ou superiores $^{(26,28)}$ a outros estudos. Acredita-se que os valores relativamente elevados de adiposidade subcutânea encontrados possam ser explicados, pelo menos em parte, pelo nível socioeconômico dos participantes. Infelizmente, essa hipótese não pôde ser confirmada pela inexistência de crianças de diferentes classes socioeconômicas na amostra investigada.

Com relação à distribuição da gordura corporal, os resultados indicam que a gordura subcutânea da região central e das extremidades do corpo foi relativamente estável em todos os grupos etários, bem como em ambos os sexos. Apesar disso, foi possível verificar uma quantidade de gordura subcutânea mais elevada na região tricipital, o que de certa forma corrobora os dados produzidos por outros estudos disponíveis na literatura(26,29).

Os resultados indicaram, também, a existência de um número maior de meninas $(\sim 15 \%)$ com níveis de gordura corporal abaixo do desejável quando comparados aos meninos ( 3\%). Este fato chama a atenção, uma vez que quantidade extremamente baixa de gordura corporal pode estar associada a algumas desordens nutricionais como a aneroxia nervosa, principalmente entre adolescentes do sexo feminino e a desnutrição protéico-energética afetando o processo de crescimento físico(32-33).

Outro aspecto importante desse estudo foi à quantidade elevada de crianças que apresentaram excesso de gordura corporal ( $28 \%$ para os meninos e $\sim 14 \%$ para as meninas). Esses resultados são bastante preocupantes, uma vez que valores de gordura corporal relativa superiores a $25 \%$ e $30 \%$, em meninos e meninas, respectivamente, estão associados a um alto risco de desen- volvimento de doenças cardiovasculares, hipertensão, hipercolesterolemia e hiperlipidemia ${ }^{(2-3)}$. Além disso, os resultados encontrados foram bastante superiores ao relatados por outros estudos no Brasil(133-34), sugerindo uma forte associação desses resultados com as características econômicas e culturais da amostra(36)

Apesar da atividade física habitual e da ingestão energética não ter sido controlada neste estudo, acredita-se que o pequeno envolvimento em atividades físicas vigorosas e/ou a ingestão de alimentos de alto valor energético possam ser os principais determinantes desse fenômeno, sobretudo em crianças e adolescentes.

Um outro fator que pode ter comprometido, pelo menos em parte, alguns dos resultados do presente estudo foi a falta de controle do estado maturacional das crianças investigadas.

Vale ressaltar que o método de espessura de dobras cutâneas, embora seja de fácil aplicação, de baixo custo operacional, de possibilitar a avaliação de um número elevado de indivíduos e de apresentar erro de medida aceitável, apresenta limitações importantes e que não devem ser desprezadas no momento da interpretação dos resultados, sobretudo quando aplicado em populações jovens $^{(37)}$

\section{CONCLUSÕES}

Os resultados do presente estudo indicam que, aparentemente, não foi possível identificar a presença de dimorfismo sexual, tanto para a quantidade quanto para a distribuição da gordura corporal. Por outro lado, um acréscimo progressivo de adiposidade corporal e de massa corporal magra foi encontrado como o avançar da idade, em crianças de ambos os sexos.

Além disso, as informações desse estudo apontaram um elevado contingente de crianças, de ambos os sexos, com quantidade excessiva de gordura corporal relativa, o que pode favorecer o aumento do risco de desenvolvimento de sobrepeso/obesidade e, conseqüentemente, de disfunções metabólicas e comportamentais associadas a esse fenômeno.

Os resultados sugerem a necessidade da realização de estudos que adotem um delineamento longitudinal, de preferência com a utilização de grupos que apresentem características socioeconômicas, étnicas e culturais distintas e com o controle nutricional, do nível de atividade física habitual e dos processos maturacionais, para a verificação das possíveis modificações individuais ao longo do tempo e os períodos sensíveis nos quais essas modificações se processam.

\section{AGRADECIMENTOS}

Os autores agradecem ao CNPq e a CAPES, pelo auxílio financeiro concedido na forma de bolsas de estudo e de produtividade em pesquisa, e a inesquecível companheira Alexandra Ramos Rodrigues (in memorium), por sua contribuição na execução deste trabalho.

Todos os autores declararam não haver qualquer potencial conflito de interesses referente a este artigo.

\section{REFERÊNCIAS}

1. Lohman TG. The use of skinfolds to estimate body fatness on children and youth JOPERD. 1987;58:98-102.

2. Twisk JWR, Kemper HCG, Mechelen W, Post GB, Lenth FJ. Body fatness: Iongitudinal relationship of body mass index and the sum of skinfolds with other risk factors for coronary heart disease. Int J Obes Relat Metab Disord. 1998;22:915 22

3. Willians DP, Going SB, Lohman TG, Harsha DW, Srinivasan SR, Webber LS, et al. Body fatness and risk for elevated blood pressure, total cholesterol, and serum lipoprotein ratios in children and adolescents. Am J Public Health. 1992;82:35863

4. Hill JA, Peters JC. Environment contributions to the obesity epidemic. Science. 1998;280:1371-4. 
5. Dietz WH. Childhood weight affects adult morbidity and mortality. J Nutr. 1998; 128:411S-4S.

6. Dietz WH. The role of lifestyle in health: the epidemiology and consequences of inactivity. Proc Nutr Soc. 1996;55:621-3.

7. Goran MI. Measurements issues related to studies of childhood obesity: assessment of body composition, body fat distribution, physical activity and food intake. Pediatrics. 1998;101:505-18

8. Dwyer T, Blizzard CL. Defining obesity in children by biological endpoint rather than population distribution. Int J Obes Relat Metab Disord. 1996;20:472-80.

9. Daniels RS, Morrison JA, Sprecher DL, Khoury P, Kimball TR. Association of body distribution and cardiovascular risk factors in children and adolescents. Circulation. 1999;99:541-5.

10. Guedes DP, Guedes JERP. Distribuição da gordura corporal, pressão arterial e níveis de lipídios-lipoproteínas plasmáticas. Arq Bras Cardiol. 1998;70:93-8.

11. Vanhala M, Vanhala P, Kumpusalo E, Kalonen P, Takala J. Relation between obesity from childhood to adulthood and the metabolic syndrome: population based study. BMJ. 1998;317:319-20.

12. Malcom G, Oalmann M, Strong J. Risk factors for atherosclerosis in young subjects: the PDAY Study. Ann N Y Acad Sci. 1997;817:179-88.

13. Must A. Morbidity and mortality associated with elevated body weight in children and adolescents. Am J Clin Nutr. 1996;63:445S-7S.

14. Barlow SE, Dietz WH. Obesity evaluation and treatments: expert committee recommendations. Pediatrics. 1998;102:1-19.

15. Ross WD, Marfell-Jones MJ. Kinanthropometry. In: MacDougall JD, Wenger HA Green HS, editors. Physiological testing of the elite athlete. New York: Mouvement Publications; 1982. p. 75-115

16. Eveleth $\mathrm{PH}$, Tanner JM. Worldwide variation in human growth. $2^{\text {nd }}$ ed. Cambridge: Cambridge University Press; 1990.

17. Almeida PM, Wickerhauser H. O critério ABA-ABIPEME: em busca de uma atualização. São Paulo; 1991.

18. Gordon CC, Chumlea WC, Roche AF. Stature, recumbent length, and weight. In: Lohman TG, Roche AF, Martorell R, editors. Anthropometric standardization reference manual. Champaign: Human Kinetics Books; 1988. p. 3-8.

19. Harrison GG, Buskirk ER, Carter LJE, Johston FE, Lohman TG, Pollock ML, et al. Skinfold thicknesses and measurement technique. In: Lohman TG, Roche AF, Martorell R, editors. Anthropometric standardization reference manual. Champaign: Human Kinetics Books; 1988. p. 55-70

20. Slaughter MH, Lohman TG, Boileau RA, Horswill CA, Stillman RJ, Van Loan MD et al. Skinfold equations for estimation of body fatness in children and youth. Hum Biol. 1988;60:709-23.

21. Monyeki MA, Koppes LLJ, Kemper HCG, Monyeki KD, Toriola AL, Pienaar AE, et al. Body composition and physical fitness of undernourished South African rural primary school children. Eur J Clin Nutr. 2005;59:877-83.
22. Abrantes MM, Lamounier JA, Colosimo EA. Prevalência de sobrepeso e obesidade nas regiões Nordeste e Sudeste do Brasil. Rev Assoc Med Brasil. 2003;49:1626.

23. Cole TJ, Bellizzi MC, Flegal KM, Dietz WH. Establishing a standard definition for children overweight and obesity worldwide: international survey. BMJ. 2000;302:16 .

24. Hooper CA, Gruber MB, Munoz KD, MacConnie SE, Pfingston YM, Nguyen K. Relationship of blood cholesterol to body composition, physical fitness, and dietary intake measures in third-grade children and their parents. Res Q Exerc Sport. 2001;72:182-8.

25. Fulton JE, McGuire MT, Caspersen CJ, Dietz WH. Interventions for weight loss and weight gain prevention among youth. Sports Med. 2001;31:153-65.

26. Böhme MTS. Aptidão física e crescimento físico de escolares de 7 a 17 anos de Viçosa, MG. Rev Min Educ Fís. 1996:4:45-60

27. Gonçalves HR. Aspectos antropométricos e motores em escolares de 7 a 14 anos de alto nível socioeconômico. Rev Assoc Prof Educ Fís. 1995;10:71-80

28. Guedes DP, Guedes JERP. Composição corporal em crianças e adolescentes do município de Londrina - Paraná. Rev Assoc Prof Educ Fís. 1995;10:3-15.

29. Mechelen WV, Kemper HCG. Body growth, body composition, and physical fitness. In: Kemper HCG, editor. The Amsterdam Growth Study: a longitudinal analysis of health, fitness and lifestyle. Champaign: Human Kinetics Books; 1995. p. 52-85.

30. Center for Disease Control and Prevention - National Center for Health Statistics, 2002. CDC Growth Charts: UNITED STATES [on-line]. Available from: http:// www.cdc.gov/growthcharts/, [maio, 15, 2004].

31. Malina RM, Bouchard C, Bar-Or O. Growth, maturation and physical activity. $2^{\text {nd }}$ ed. Champaign: Human Kinetics Books; 2004.

32. Kerruish KP, O'Connor J, Humphries IRJ, Kohn MR, Clarke SD, Briody JN, et al. Body composition in adolescents with anorexia nervous. Am J Clin Nutr. 2002; 75:31-7.

33. Probst M, Goris M, Vandereycken W, Coppenolle HV. Body composition of anorexia nervous patients assessed by underwater weighing and skinfold-thickness measurements before and after weight gain. Am J Clin Nutr. 2001;73:190-7.

34. Pires EAG, Pires MC, Petroski EL. Adiposidade corporal, padrão de comportamento e estresse em adolescentes. Rev Bras Cineantropom Desempenho Hum. 2002;4:7-16.

35. Guedes DP, Guedes JERP. Prevalência de sobrepeso e obesidade em crianças e adolescentes do município de Londrina (PR), Brasil. Motriz. 1998;4:18-25.

36. Ronque ERV, Cyrino ES, Dórea VR, Serassuelo Jr H, Galdi EHG, Arruda M. Prevalência de sobrepeso e obesidade em escolares de alto nível socioeconômico em Londrina, Paraná, Brasil. Rev Nutr. 2005;18:709-17.

37. Sopher A, Shen W, Pietrobelli A. Pediatric body composition methods. In: Heymsfield SB, Lohman TG, Wand Z, Going SB, editors. Human body composition. $2^{\text {nd }}$ ed. Champaign: Human Kinetics Books; 2005. p. 129-39. 